\title{
FREE COVERINGS AND MODULES OF BOUNDARY LINKS
}

BY

\author{
NOBUYUKI SATO
}

\begin{abstract}
Let $L=\left\{K_{1}, \ldots, K_{m}\right\}$ be a boundary link of $n$-spheres in $S^{n+2}$, where $n>3$, and let $X$ be the complement of $L$. Although most of the classical link invariants come from the homology of the universal abelian cover $\tilde{X}$ of $X$, with increasing $m$ these groups become difficult to manage. For boundary links, there is a canonical free covering $X_{\omega}$, which is simultaneously a cover of $\tilde{X}$. Thus, knowledge of $H_{*} X_{\omega}$ yields knowledge of $H_{*} \tilde{X}$. We study general properties of such covers and obtain, for $1<q<n / 2$, a characterization of the groups $H_{q} X_{\omega}$ as modules over the group of covering transformations. Some applications follow.
\end{abstract}

1. Introduction. A link of codimension two is a collection $L=\left\{K_{1}, \ldots, K_{m}\right\}$ of disjointly embedded smooth $n$-spheres in $S^{n+2}$. When the $K_{i}$ are the boundaries of smooth, pairwise disjoint, oriented submanifolds $V_{i}^{n+1}$ of $S^{n+2}$, we say that $L$ is a boundary link. If $N\left(K_{i}\right) \approx K_{i} \times D^{2}$ is a tubular neighborhood of $K_{i}$ in $S^{n+2}$, then the space $X=\mathrm{Cl}\left\{S^{n+2}-\cup_{i} N\left(K_{i}\right)\right\}$ is an $(n+2)$-manifold with boundary. It is called the complement of $L$.

Many of the classical link invariants come from the homology of the universal abelian cover $\tilde{X}$ of $X$. These groups are modules over $\Lambda=\mathbf{Z}\left[\mathbf{Z}^{m}\right]$, where $\mathbf{Z}^{m}$ is the free abelian group of rank $m$ via the covering transformations. Unfortunately, $\Lambda$-module theory increases in difficulty with $m$ (at least). However, for boundary links, there is another covering space which arises naturally, which is also a covering of $\tilde{X}$. Thus, knowledge about the homology of this space yields information about $H_{*} \tilde{X}$.

Let $\mu_{1}, \ldots, \mu_{m}$ be the elements of $\pi_{1} X$ represented by $* \times \partial D^{2}$ in $K_{i} \times D^{2}$ for each $i$, oriented so that this circle links $K_{i}$ with linking number +1 . The $\mu_{i}$ are called the meridians of $L$. The following result of Smythe [14] and Gutierrez [3] gives a necessary and sufficient condition for the link to be boundary.

LEMMA 1.1. A link of $m$ components is a boundary link if and only if there exists a surjection of $\pi_{1} X$ onto the free group $F_{m}$ on $m$ generators $x_{1}, \ldots, x_{m}$ carrying the meridians $\mu_{i}$ to the $x_{i}$.

Let $X_{\omega}$ be the regular covering of $X$ associated to the kernel of this map. It is called the free cover of $X$ and its homology groups $H_{q} X_{\omega}$ are left $\Phi=Z\left[F_{m}\right]-$ modules via the covering transformations.

We will make a study of the ring $\Phi$ and characterize a certain kind of $\Phi$-module called type $L$. These modules arise in the study of free covers over spaces which are

Received by the editors July 26, 1978 and, in revised form, November 29, 1978 and March 27, 1980. AMS (MOS) subject classifications (1970). Primary 57C45. 
homologically an $m$-fold wedge of circles. We use these modules of type $L$ to give a complete characterization of the $\Phi$-modules $H_{q} X_{\omega}$ in the range $1<q<n / 2$, $n \geqslant 3$. As applications of this result, we obtain a generalization of a result of Kervaire [7, Theorem II.1] and a sufficient condition for a polynomial in $m$-variables to be an Alexander polynomial of a high-dimensional link (actually, it can be shown [12] that this is also a necessary condition for high-dimensional boundary links).

Some of the results in this paper (including the main result) form part of the author's Ph.D. thesis, which was written at Brandeis University under the direction of Jerome Levine and presented in April, 1978. We wish to thank Professor Levine for much help and encouragement. We also thank Hyman Bass, who pointed out part (iii) of Theorem 2.1 and the remark after Corollary 2.2.

The results of $\S 3$ are generalizations to links of certain results of [9]. See also [6] for related results.

2. The ring $\Phi=\mathrm{Z}\left[F_{m}\right]$ and free covers. For $m>1, \Phi$ is neither noetherian nor commutative. However, $\Phi$ has certain properties which simplify its module theory.

THEOREM 2.1. Let $\Phi=\mathbf{Z}\left[F_{m}\right]$. Then

(i) $\Phi$ has no zero-divisors.

(ii) $\Phi$ has global dimension two.

(iii) $\Phi$ is coherent, that is, the kernel of any homomorphism between finitely presented modules is again finitely presented.

(iv) Finitely generated projective $\Phi$-modules are free.

(v) There is an augmentation map $\varepsilon: \Phi \rightarrow \mathbf{Z}$ which sends $\sum n_{g} g$ to $\sum n_{g}$. The kernel of $\varepsilon$ is a free module of rank $m$, with basis $\left\{x_{1}-1, \ldots, x_{m}-1\right\}$ where $x_{1}, \ldots, x_{m}$ are the free generators of $F_{m}$.

Part (i) is due to [4]. Part (ii) follows from a very general categorical result [11, Theorem 31.2]. Part (iii) is due to [15]. Part (iv) comes from [1]. For a proof of part (v), see [5, p. 196].

From part (iii), we have the following corollary.

Corollary 2.2. Let $C=\left\{C_{q}, \partial_{q}\right\}$ be a free left chain complex over $\Phi$ of finite type. For example, let $C$ be the cellular chain complex of a free covering of $a$ $C W$-complex $Y$ with finitely many cells in each dimension. Then for each $q, H_{q} C$ is a finitely presented left $\Phi$-module.

REMARK. Theorem 2.2 parts (ii)-(iv) actually imply that the kernel of any homomorphism between free $\Phi$-modules of finite rank is in fact a free module of finite rank. If $Z_{q}=\operatorname{ker} \partial_{q}$ in the above, then a canonical finite presentation of $H_{q} C$ is given by

$$
C_{q+1} \stackrel{\partial_{q+1}}{\rightarrow} Z_{q} \rightarrow H_{q} C \rightarrow 0
$$

We will see that $\Phi$-modules with certain seemingly strange properties play a crucial role. 
Definition. A $\Phi$-module $M$ is said to be of type $L$ if

(i) $M$ is a finitely presented $\Phi$-module, and

(ii) $\operatorname{Tor}_{q}^{\Phi}(\mathbf{Z}, M) \cong 0$ for all $q$, where $\mathbf{Z}$ is regarded as a right $\Phi$-module with trivial action via the augmentation map $\varepsilon$.

RemarK. Condition (ii) is equivalent to the condition that the homology of $F_{m}$ with twisted coefficients in $M$ is zero in all dimensions. In fact, $\operatorname{Tor}_{q}^{\Phi}(\mathbf{Z}, M) \cong$ $H_{q}\left(F_{m} ; M\right)$ and the isomorphism is often taken as the definition of group homology.

We now characterize modules of type $L$ in such a way that we will be able to construct many examples easily, showing that the definition is far from empty.

Proposition 2.3. Let $M$ be a finitely presented left $\Phi$-module. The following are equivalent.

(i) $M$ is of type $L$.

(ii) The map $\bigoplus_{i=1}^{m} M \rightarrow M$ given by $\left(t_{1}, \ldots, t_{m}\right) \mapsto \Sigma\left(x_{i}-1\right) t_{i}$ is an isomorphism.

(iii) $M$ has a free resolution

$$
0 \rightarrow \Phi_{\left(a_{i j}\right)}^{r} \underset{\left(b_{k l}\right)}{\rightarrow} \Phi^{t} \rightarrow M \rightarrow 0
$$

with the $a_{i j}$ and $b_{k l}$ in $\Phi$, such that

(a) $r+t=s$.

(b) The greatest common divisor of the $r \times r$ minor determinants of the integral matrix $\left(\varepsilon\left(a_{i j}\right)\right)$ is 1 .

(c) The greatest common divisor of the $t \times t$ minor determinants of the integral matrix $\left(\varepsilon\left(b_{k l}\right)\right)$ is 1 .

Proof. Since $M$ is finitely presented, we need only check that (ii) and (iii) are equivalent to $\operatorname{Tor}_{q}^{\Phi}(\mathbf{Z}, M) \cong 0$ for all $q$. But (ii) is a reformulation of this condition using the canonical free resolution of $\mathbf{Z}$ given by Theorem 2.1 part (v). Any finitely presented module $M$ has a short free resolution as in (iii), and it is easily verified that conditions (a)-(c) are necessary and sufficient conditions for the complex of abelian groups formed by tensoring this free resolution with $\mathbf{Z}$ to be exact, either directly or by applying the theory in [2].

AN EXAMPLe. Let $g$ be any nonunit in $\Phi$ such that $\varepsilon(g)= \pm 1$, for example, $g=2 x_{1}-1$. By part (i) of Theorem $2.1, g$ is not a zero-divisor so that the module $M$ defined by the following sequence is of type $L$ :

$$
0 \rightarrow \Phi \underset{g}{\rightarrow} \Phi \rightarrow M \rightarrow 0
$$

This construction extends easily to the cokernel of any diagonal matrix over $\Phi$ whose diagonal entries satisfy the conditions on $g$ above.

The relevance of the concept of type $L$ is seen in the following result. Let $Y$ be a CW-complex with finitely many cells in each dimension, and suppose $Y$ has a free cover $Y_{\omega}$. 
Proposition 2.4. (i) $H_{q} Y_{\omega}$ is of type $L$ for all $q>0$ if and only if $H_{*} Y \approx$ $H_{*}\left(\bigvee_{i=1}^{m} S^{1}\right)$.

(ii) If $Y$ has the homology of an $m$-fold wedge of circles through dimension $n$, then $H_{q} Y_{\omega}$ is of type $L$ for $0<q<n$.

Proof. One can see this using the Cartan-Leray-Serre spectral sequence of the covering $X_{\omega} \rightarrow X$, or alternatively, one has the short exact sequence of chain complexes

$$
0 \rightarrow \bigoplus_{i=1}^{m} C_{*} X_{\omega} \rightarrow C_{*} X_{\omega} \stackrel{P_{*}}{\rightarrow} C_{*} X \rightarrow 0, \quad\left(t_{1}, \ldots, t_{m}\right) \mapsto \sum\left(x_{i}-1\right) t_{i}
$$

The proposition follows easily by analyzing the resulting long exact homology sequence as in [10] using the characterization of type $L$ given in Proposition 2.3(ii).

3. Modules of boundary links. We now specialize to the case where $X$ is the complement of a boundary link $L=\left\{K_{1}, \ldots, K_{m}\right\}$ of dimension $n \geqslant 3$. Let $X_{\omega}$ be the free covering of $X$. The main result is the following.

THEOREM 3.1. (i) $H_{k} X_{\omega}$ is a $\Phi$-module of type $L$ for $0<k<n$.

(ii) Let $A_{2}, \ldots, A_{q}$ be any sequence of $\Phi$-modules of type $L$. There exists a boundary link $L$ of dimension $n=2 q+1$ such that, if $X$ is the complement and $X_{\omega}$ is the free cover, then $H_{k} X_{\omega} \cong A_{k}$ for $1<k \leqslant q$. If $A_{q}$ has projective dimension one, then we may take $n=2 q$.

Note that part (i) follows from Proposition 2.4, since $X$ has the homology of $\bigvee_{i=1}^{m} S^{1}$ through dimension $n$ by Alexander duality. Part (ii) will follow from a method analogous to that in $[9, \S \S 9$ and 10], which goes back to unpublished work of C. T. C. Wall. We begin by constructing CW-complexes with the desired homological properties.

Lemma 3.2. Let $A$ be any $\Phi$-module of type $L$ and let $q>1$. Then there exists $a$ finite $C W$-complex $P$ of dimension $q+2$ such that

(i) $\pi_{1} P \cong F_{m}$.

(ii) $H_{*} P \approx H_{*}\left(\bigvee_{i=1}^{m} S^{1}\right)$.

(iii) If $P_{\omega}$ is the universal cover of $P$, then $H_{k} P_{\omega} \cong 0$ unless $k=0$ or $q$, and $H_{q} P_{\omega} \cong A$.

(iv) The 1-skeleton of $P$ is $\bigvee_{i=1}^{m} S^{1}$. If $A$ has projective dimension one, we can choose $P$ to be $(q+1)$-dimensional.

The proof is analogous to the proof of [9, Lemma 9.4].

Lemma 3.3. Let $A_{2}, \ldots, A_{q}$ be any sequence of $\Phi$-modules of type $L$. Then there exists a finite $C W$-complex $P$ of dimension $q+2$ such that

(i) $\pi_{1} P \cong F_{m}$.

(ii) $H_{*} P \cong H_{*}\left(\bigvee_{i=1}^{m} S^{1}\right)$.

(iii) If $P_{\omega}$ is the universal cover of $P$, then $H_{k} P_{\omega} \cong 0$ for $k>q$ and $H_{k} P_{\omega} \cong A_{k}$ for $1<k \leqslant q$.

(iv) The 1-skeleton of $P$ is $\bigvee_{i=1}^{m} S^{1}$. If $A_{q}$ has projective dimension one, then we can choose $P$ to be $(q+1)$-dimensional. 
Proof. For each $k=2, \ldots, q$ let $W_{k}$ be that complex of dimension $k+2$ constructed by Lemma 3.2. The complex $P$ is formed from the disjoint union of the $W_{k}$ by identifying their 1 -skeletons. It is easily verified that $P$ has the correct homological properties.

The idea now is to make $P$ into a handlebody, and show that this handlebody is in fact the complement of a boundary link. Let $d=\operatorname{dim} P$. Note that $d \geqslant 3$.

Proposition 3.4. $P$ embeds in $\mathbf{R}^{n+3}$, where $n \geqslant 2 d-3$. Let $Y$ be the boundary of a regular neighborhood $N(P)$. Then the composite map

$$
Y \underset{\text { inclusion }}{\rightarrow} N(P) \underset{\text { retraction }}{\rightarrow} P
$$

is $(n+2-d)$-connected. $Y$ has nonzero homology only in dimension $0,1, n+1$ and $n+2$.

For a proof, see [9, Lemma 10.1]. Let $\varphi_{1}, \ldots, \varphi_{m}: S^{1} \times D^{n+1} \rightarrow Y$ be embeddings with disjoint images representing the free generators of $\pi_{1} Y \cong F_{m}$, and let $X=\mathrm{Cl}\left\{Y-\cup_{i}\right.$ im $\left.\varphi_{i}\right\}$. By general position, the pair $(Y, X)$ is $n$-connected. Performing surgery on these maps yields a manifold $\Sigma^{n+2}$ and embeddings $\psi_{1}, \ldots, \psi_{m}: D^{2} \times S^{n} \rightarrow \Sigma$ with disjoint images. It is not difficult to check that $\Sigma$ is a simply connected homology $(n+2)$-sphere, and hence a homotopy sphere. Since $n+2 \geqslant 5, \Sigma$ is in fact a PL sphere, by arguments of Smale [13]. Hence, we may change the differentiable structure of $\Sigma$ at a point not on the image of the $\psi_{i}$ to obtain the standard sphere $S^{n+2}$. Let $L$ be the link defined by taking

$$
K_{i}=\psi_{i}\left(0 \times S^{n}\right) .
$$

Then the complement of $L$ is $X$. By construction, $\pi_{1} X$ is the free group generated by the meridians, so that by Lemma $1.1, L$ is a boundary link. The case when $A_{q}$ has projective dimension one is done by embedding $P^{q+1}$ in $\mathbf{R}^{n+3}$ where $n=2 q$. This completes the proof of 3.1 .

4. Some applications. We now mention two applications of the main result. The first is a generalization of a theorem of Kervaire.

Corollary 4.1. Suppose that $L$ is an $n$-dimensional link of $m$ components, $n \geqslant 3$, with complement $X$. Suppose that $X$ satisfies, for some $q, 1<q<n$,

$$
\pi_{k} X \cong \pi_{k}\left(\bigvee_{i=1}^{m} S^{1}\right) \text { for } 0 \leqslant k<q .
$$

Then $\pi_{q} X$, as a module over $\mathrm{Z}\left[\pi_{1} X\right] \cong \Phi$, is of type L. Conversely, if $A$ is any $\Phi$-module of type $L$ and $q>1$, there exists a boundary link $L$ satisfying (*) such that $\pi_{q} X \cong A$.

REMARK. Kervaire [7, Theorem II.1] proved this result when $m=1$ (for knots).

Proof. One needs only to remark that under hypothesis (*), $\pi_{q} X \cong H_{q} X_{\omega}$. Theorem 3.1 now applies to prove this result.

The next application requires some introduction. As for knots, the Alexander polynomials of links are the elementary divisors of the homology groups of the universal abelian cover of the complement (see [8]). Briefly, the group of covering 
transformations of the universal abelian cover $\tilde{X}$ of $X$ is the free abelian group $\mathbf{Z}^{m}$ of rank $m$. Thus, the groups $H_{*} \tilde{X}$ are finitely generated modules over $\Lambda=\mathbf{Z}\left[\mathbf{Z}^{m}\right]$, which we identify with the localized polynomial ring $\mathbf{Z}\left[x_{1}, \ldots, x_{m}, x_{1}^{-1}, \ldots, x_{m}^{-1}\right]$ by writing $\mathbf{Z}^{m}$ multiplicatively. $\Lambda$ is a noetherian commutative ring. For each group $H_{q} \tilde{X}$, take a $k \times l$ presentation matrix for $H_{q} \tilde{X}$ as a $\Lambda$-module. We may assume that $k$ (the number of relations) exceeds the number of generators $l$. The elementary divisors $\Delta_{i}^{q}$ of $H_{q} \tilde{X}$ are the greatest common divisors of the $(l-i) \times(l-i)$ minor determinants of the presentation matrix. It is not difficult to see that they are independent of the choice of matrix, and are defined up to units of $\Lambda$. Since elements of $\Lambda$ are all polynomials up to this sort of equivalence, we call the collection $\left\{\Delta_{i}^{q}\right\}$ the Alexander polynomials of the link. It is clear (by the process of expansion by minors) that $\Delta_{i+1}^{q} \mid \Delta_{i}^{q}$ for each $i, q$.

Theorem 3.1 applies here to yield a sufficient condition for a sequence of polynomials $p_{i}^{q}\left(x_{1}, \ldots, x_{m}\right)$ to be the Alexander polynomials of a boundary link of dimension $n$, for a range of $q$.

Corollary 4.2. Let $p_{i}^{q}\left(x_{1}, \ldots, x_{m}\right), i=1, \ldots, k, q=2, \ldots,[n / 2]$, be any sequence of polynomials satisfying

(i) $p_{i+1}^{q} \mid p_{i}^{q}$ for each $i, q$,

(ii) $p_{i}^{q}(1,1, \ldots, 1)= \pm 1$ for all $i, q$.

Then the $p_{i}^{q}$ are the $\Delta_{i}^{q}$ for some $n$-dimensional boundary link, for the values of $q$ specified above.

Proof. Let $\lambda_{i}^{q}=p_{i}^{q} / p_{i+1}^{q}$ for each $q, i=0, \ldots, k-1$, and let $\lambda_{k}^{q}=p_{k}^{q}$. Then for each $i, q, \lambda_{i}^{q}(1,1, \ldots, 1)= \pm 1$. We will construct an $n$-dimensional boundary link such that, if $\tilde{X}$ is the universal abelian cover of the complement, $H_{q} \tilde{X}$ has the diagonal matrix $\left(\lambda_{0}^{q}, \ldots, \lambda_{k}^{q}\right)$ as presentation matrix. Then this link will satisfy the conclusion of the theorem.

The first step is to lift the diagonal matrices $\left(\lambda_{0}^{q}, \ldots, \lambda_{k}^{q}\right)$ to $\Phi$. This may be done, for example, by imagining that the variables no longer commute in the polynomials $\lambda_{i}^{q}$. We apply Theorem 3.1 to realize the $\Phi$-modules presented by these matrices. It is easy to check that, in $\tilde{X}$, we are in effect realizing the $\Lambda$-modules presented by the original matrices. This completes the argument.

As remarked before, one can show that the above sufficient condition can be shown to be necessary for boundary links (see [12]). In that work, we also obtain results for $H_{1} \tilde{X}$, and $H_{q} \tilde{X}$ when $n=2 q-1$, cases which are not covered by the techniques of this work.

\section{REFERENCES}

1. H. Bass, Projective modules over free groups are free, J. Algebra 1 (1964), 367-373.

2. D. A. Buchsbaum and D. Eisenbud, What makes a complex exact?, J. Algebra 25 (1973), 259-268.

3. M. Gutierrez, Boundary links and an unlinking theorem, Trans. Amer. Math. Soc. 171 (1972), 491-499.

4. G. Higman, The units of group rings, Proc. London Math. Soc. 46 (1940), 231-248.

5. P. Hilton and U. Stammbach, $A$ course in homological algebra, Springer-Verlag, Berlin and New York, 1971.

6. P. Hirschhorn, Link complements and coherent group rings, Illinois J. Math. 24 (1980), 159-163.

7. M. Kervaire, Les noeds de dimensions superieures, Bull. Soc. Math. France 93 (1965), 225-271. 
8. J. Levine, Polynomial invariants of knots of codimension two, Ann. of Math. (2) 84 (1966), 537-554.

9. Knot modules. I, Trans. Amer. Math. Soc. 229 (1977), 1-50.

10. J. Milnor, Infinite cyclic coverings, Topology of Manifolds (J. Hocking, ed.), Prindle, Weber and Schmidt, Boston, Mass., 1962.

11. B. Mitchell, Rings with several objects, Advances in Math. 8 (1972), 1-161.

12. N. Sato, Algebraic invariants of boundary links, Trans. Amer. Math. Soc. (to appear).

13. S. Smale, On the structure of manifolds, Amer. J. Math. 84 (1962), 387-399.

14. N. Smythe, Boundary links, Topology Seminar (Wisconsin, 1965), (R. H. Bing and R. Bean, eds.), Princeton Univ. Press, Princeton, N. J., 1966, pp. 69-72.

15. F. Waldhausen, Whitehead groups of generalized free products, Lecture Notes in Math., vol. 342, Springer-Verlag, Berlin and New York, 1973.

Department of Mathematics, Brandeis University, Waltham, Massachusetts 02154

Department of Mathematics, University of TeXas, Austin, TeXas 78712 (Current address) 